\title{
CEREBELLAR HYPOPLASIA ASSOCIATED WITH SYSTEMIC DEGENERATION IN EARLY LIFE
}

\author{
BY \\ R. M. NORMAN and H. URICH \\ From the Burden Neuropathological Laboratory, Frenchay Hospital, Bristol
}

Hypoplasia predominantly affecting the lateral lobes of the cerebellum has on several occasions been found associated with marked underdevelopment of the griseum pontis and middle peduncle (Vogt and Astwazaturow, 1912; Brun, 1917, 1918; Brouwer, 1924; Biemond, 1955). Brouwer named this condition " hypoplasia ponto-neocerebellaris" and emphasized the fact that the same neuronal system was affected in this prenatal malformation as in olivo-ponto-cerebellar atrophy. Support was thus given to the theory already put forward by Winkler (1923) that a congenital weakness of the derivatives of the cell bands of Essick (1912) underlay the brain-stem degenerations of olivo-pontocerebellar atrophy. The two cases described in this paper take this comparison a stage further. Both are examples of cerebellar hypoplasia occurring in infants who died during the first year of life. Detailed examination of the brain-stem in the first case has revealed a much closer resemblance to olivoponto-cerebellar atrophy than has hitherto been suspected. More remarkable, however, is that in both our cases there is evidence of degeneration having taken place in certain other nuclei known to be liable to involvement in the systemic degenerations of later life. A bond has thus been disclosed linking malformation with abiotrophy.

\section{Case 1}

Clinical History.-J. L., a boy, died at the age of 5 months. The parents were second cousins. The circumstances of birth and early progress had been normal. At 2 months he was brought to a paediatrician because of the peculiar shape of the head. The mother had kept a close watch on the baby's development because his elder sister, who had died at the age of $2 \frac{1}{2}$ years, had been microcephalic and mentally defective. On examination, the sutures and the anterior fontanelle appeared to be closed or almost so. The calvaria was described as tapering towards the vertex and sloping inwards at the temples, the head circumference being $14 \frac{1}{8}$ in. It was thought that this might be a case of craniosynostosis and he was referred to the Bristol Neurosurgical Unit. The child was seen in the Outpatient Department three weeks later and the head circumference was then $14 \frac{1}{2}$ in. The retinal veins were not engorged and there seemed to be no indication for immediate operation. On admission to hospital 10 weeks later, it was confirmed that the fontanelles were closed, although the shape of the head did not conform to any of the recognized types of craniosynostosis. The child lay with the head retracted and the limbs spastic and flexed. Tonsillar herniation was suspected and it was considered to be too dangerous to carry out air studies. Parasagittal linear craniectomies were performed but the cerebral gyri were not flattened and the tension not high. Two hours after having apparently recovered from the operation the infant developed signs of respiratory failure and an emergency suboccipital decompression was carried out. It was then discovered that the cerebellum was markedly hypoplastic. The baby died during the operation.

Post-mortem examination revealed no malformation outside the cranial cavity.

Examination of the Central Nervous System in Case 1

Macroscopic.-The brain weighed $497 \mathrm{~g}$. after fixation, the cerebellum and brain-stem accounting for $20 \mathrm{~g}$. The convolutional pattern of the cerebral hemispheres was normal as were the cranial nerves and the arteries at the base. The third ventricle and the inferior horn of the left lateral ventricle were slightly dilated. The cerebellum only reached to within $3 \mathrm{~cm}$. of the occipital pole. It showed the normal anatomical arrangement of lobules but the whole organ was small and flat, the lateral lobes disproportionately so compared with the vermis and flocculi. The pons was short and the pes pontis conspicuously underdeveloped compared with the tegmentum.

Microscopic.-The cerebellum and brain-stem were serially sectioned, every fortieth section being stained for nerve cells and the adjoining section for myelin. The cerebellar cortex showed no significant lesions as regards Purkinje or granule cells. The external granular layer was of normal depth for this age. The central white matter of the lateral lobes was small in amount, poorly myelinated (Fig. 1), and in Holzer preparations showed marked fibrillary gliosis, especially in the hilum of the dentate nucleus. 


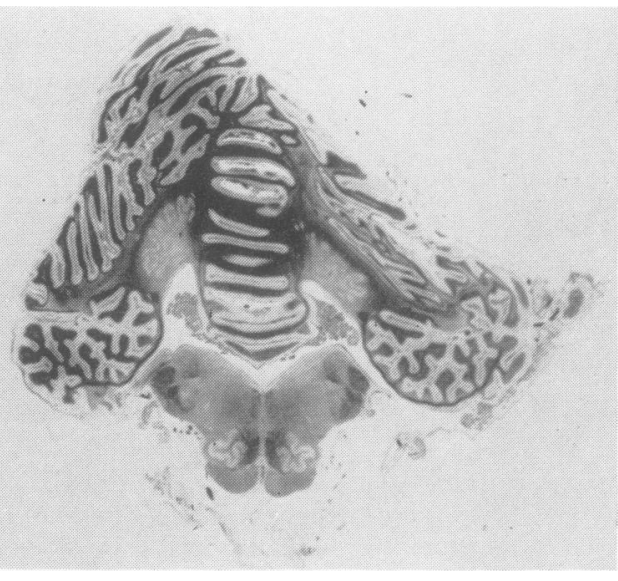

FIG. 1.-Case 1. Cerebellum: poor development of the lateral lobes compared with the vermis and particularly with the flocculi which are of normal size. The dentate nuclei are normally shaped but there is marked poverty of myelinated fibres in the hila.

Heidenhain $\times 2$

Myelination was normal in the vermis and flocculi. The dentate nuclei were severely atrophied, the remaining nerve cells tending to be grouped in small clusters (Fig. 2). These cell nests contained numerous unmyelinated axis cylinders and fewer neuroglial

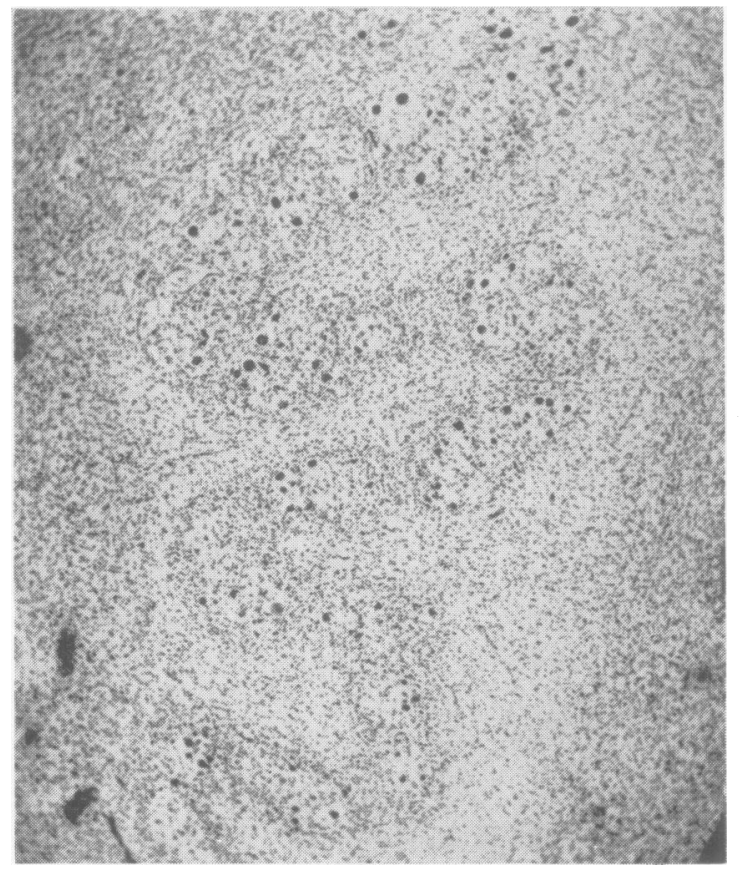

FIG. 2.-Case 1. Dentate nucleus: the majority of the nerve cells have degenerated. The original normal contour of the cell band is indicated by astrocytic nuclei.

Carbol azure $\times 30$ fibrils than the surrounding tissue. It was evident that the dentate nucleus had at one time been normally formed, since in Nissl preparations its crenated outline was clearly marked by a band of astrocytic nuclei joining together the islands of remaining nerve cells. The original shape of the nucleus was also well shown by the myelinated fibres of the amiculum. Nerve cells were more numerous in the dorsal and oral portion of the nucleus which was continuous with the nucleus emboliformis. Here and there a chromatolytic cell with a laterally displaced nucleus was seen but there was no microglial activity. The cells of the nucleus globosus were normal. The nucleus fastigii was represented on each side only by a few nerve cells placed laterally in the roof of the fourth ventricle.

Brain-stem.-The inferior olives were smaller as a whole than in the newborn. In the oral parts of the principal nucleus the dorsal and medial nerve cell band was poorly convoluted and its depth reduced in many places to a single row of irregularly spaced neurones (Fig. 3). There was a well-marked fibrillary gliosis of the hilum and capsule. The arcuate nuclei together with the external arcuate fibres and the arcuo-floccular fasciculus (stria medullaris), were absent, as was the nucleus conterminalis.

Certain nuclei situated dorso-medially in the medulla were absent or represented by a few cells only, namely, the pararaphales, paramedianu这 क dorsalis, and raphae obscurus. Another group of $\overrightarrow{0}$ nuclei normally found in the vicinity of the hypo glossal and preposital nuclei were similarly affected, namely, the intercalatus, Roller, and interfascicularis. The reticular nuclei were well developed except for the nucleus lateralis medullae which was virtually absent on both sides (Fig. 4). The pontobulbar bodies were also missing. Fibrous neuroglia was increased in the restiform bodies, the nuclear masses in the floor of the fourth ventricle and in the raphe.

The nuclei pontis were extremely poorly represented, especially at caudal levels where only a few small groups or strands of cells were present (Fig. 5). Here the pyramidal tracts were undivided into smaller bundles. At oral levels the griseum pontis was somewhat better developed and thin strands of cells partially surrounded the individual bundles of the descending cortical tracts. The great majority of these cells were small and in marked contrast to the few groups of well-developed neurones. Similar small cells could also be found in the pontine nuclei of a control case of the same age, but here they were relatively scanty. The tegmental extensions of the griseum were absent. The transverse fibres were as yet unmyelinated and in 


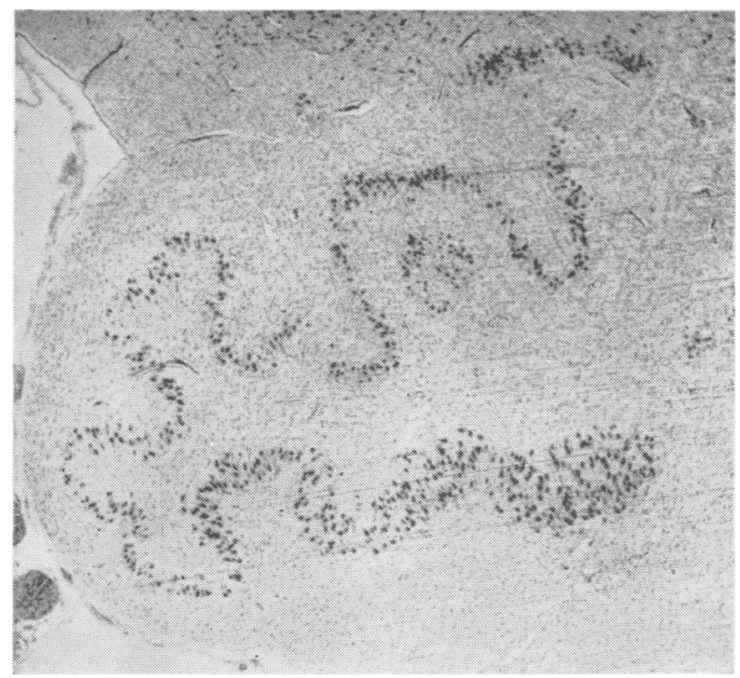

Fig. 3.-Case 1. Inferior olive: limited convolutional development and neuronal poverty especially in dorsal and lateral parts.

Carbol azure $\times 20$

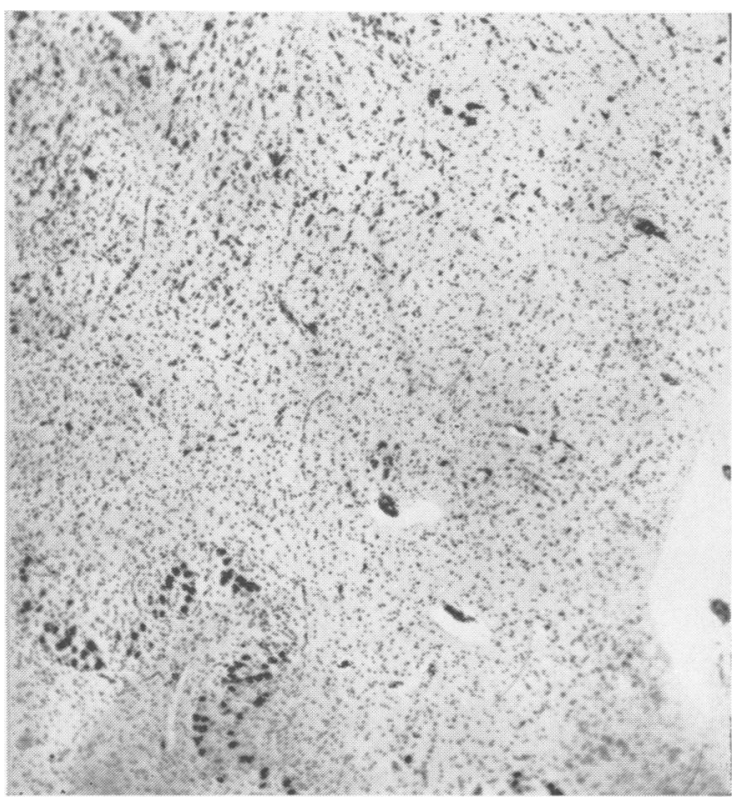

Fig. 4.-Case 1. Medulla: absence of the nucleus lateralis medullae. The dorsal folds of the inferior olive are seen below, the nucleus ambiguus above.

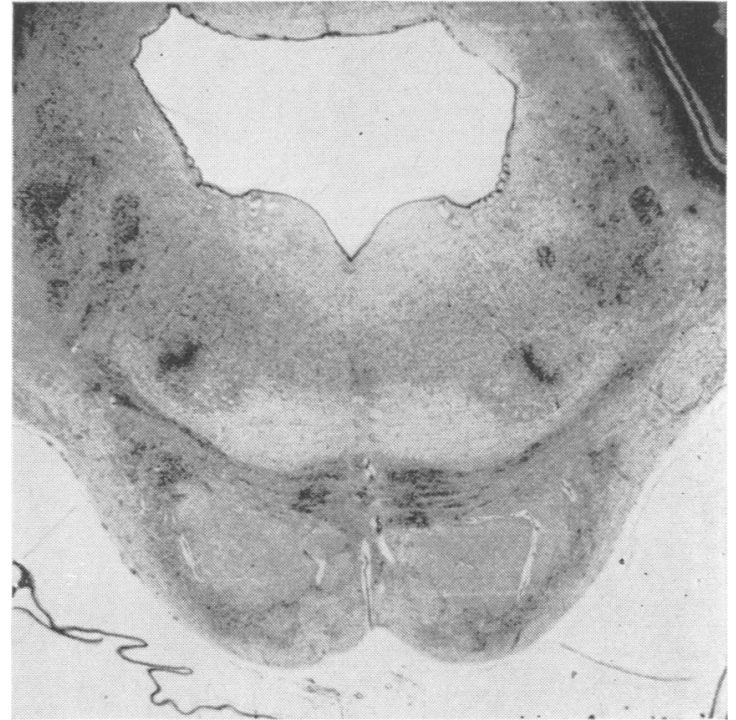

FIG. 5.-Case 1. Pons at level of superior olives: great reduction in the size of the nuclei pontis. The small pes pontis is almost filled by the pyramidal tracts.

Carbol azure $\times 6.4$

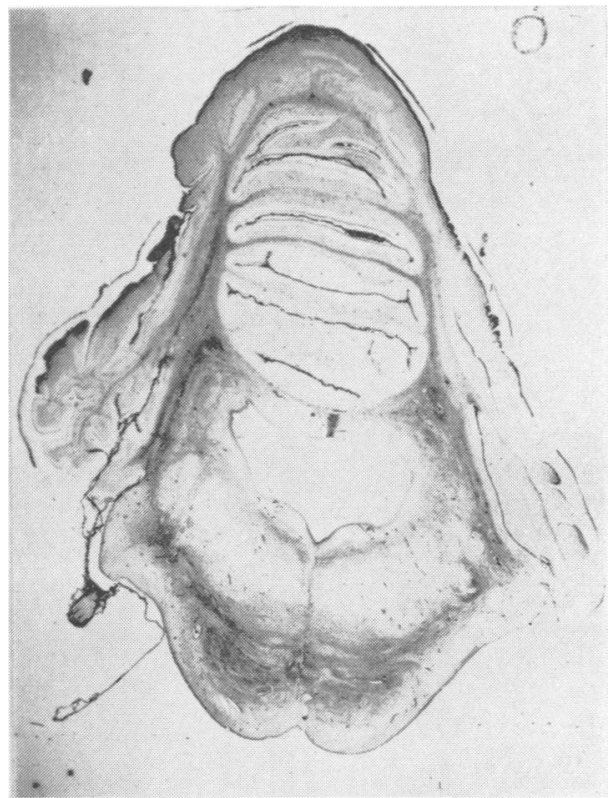

Fig. 6.-Case 1. Pons: fibrous gliosis in the pes pontis.

Holzer $\times 3.4$ 


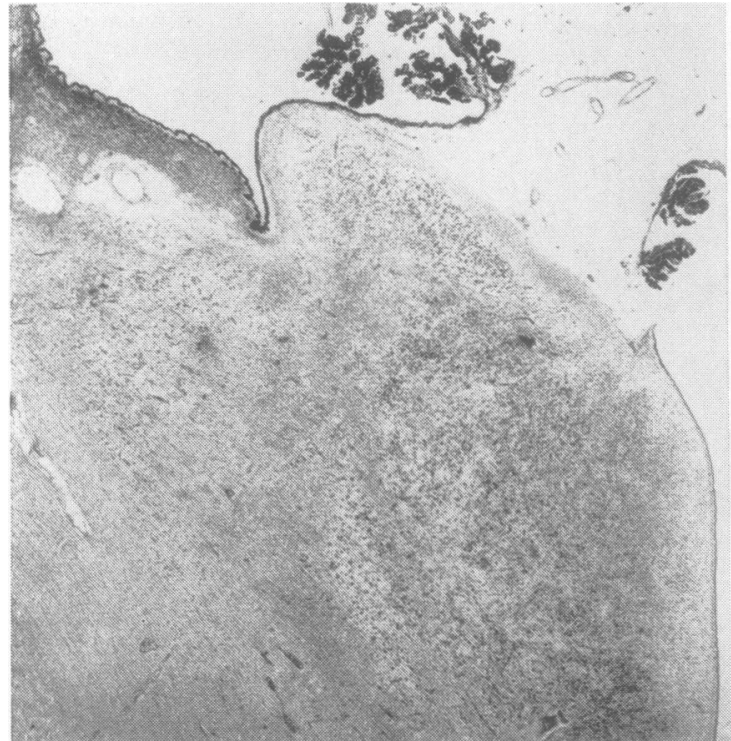

Fig. 7.-Case 1. Thalamus: atrophy of the ventro-lateral and antero-ventral nuclei. Preservation of the antero-dorsal and dorso-medial nuclei.

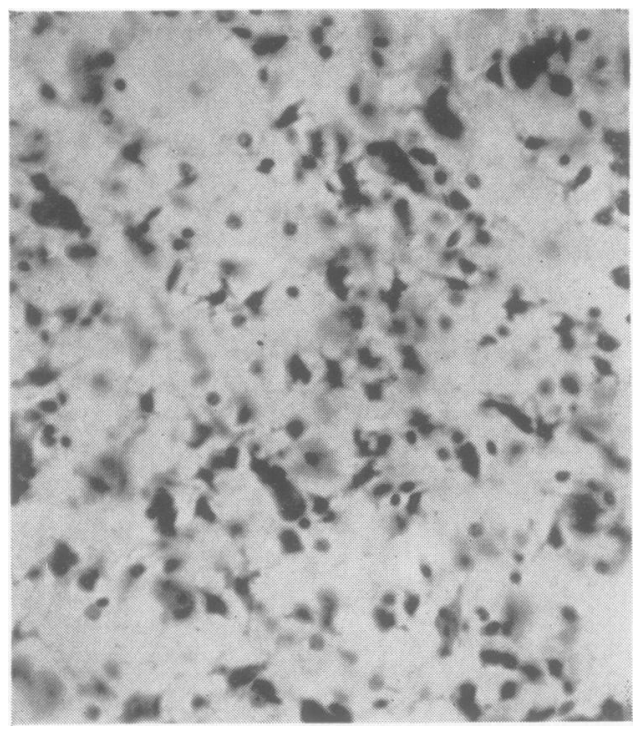

FIG. 8.-Case 1. Microglial proliferation in a degenerated part of the antero-ventral nucleus of the thalamus. Carbol azure $\times 24 \stackrel{\bar{D}}{\bar{D}} \overrightarrow{0}$

Carbol azure $\times 10$

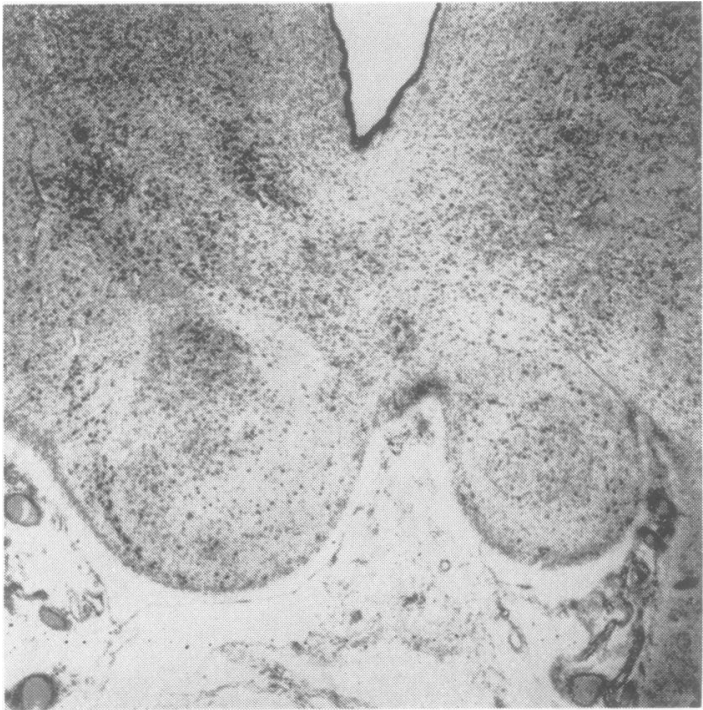

Fig. 9-Case 1. Mammillary bodies: severe bilateral loss of nerve cells in the internal nucleus.

Carbol azure $\times 16$
Gros-Bielschowsky preparations they were seen to be very poorly represented. The site of these fibres was densely gliosed (Fig. 6). Fibrillary gliosis of less intensity was present in the pyramidal tracts, griseum pontis, and medial fillet.
Thalamus.-Serial sections of the thalami showed almost complete degeneration of each nucleus ventralis lateralis. Approximately symmetrical lesions were also present in the anterior nucleus but here they were of more recent date. A considerable 
number of nerve cells had been lost in the medial and ventral parts of this nucleus, the dorsal accessory nucleus remaining intact (Fig. 7). The pathological areas contained numerous microglial cells, their thickened branches clearly visible in Nissl preparations (Fig. 8). There were no figures of neuronophagia but numerous shrunken, palely staining nerve cells were present in the vicinity of the areas of nerve cell loss.

Hypothalamus.-Severe neuronal degeneration had taken place in the medial nuclei of the mammillary bodies and very few cells remained (Fig. 9). The lateral nucleus was unaffected. There was no astrocytic gliosis but a few hyperplastic microglial cells were present. The blood vessels were normal. The bundle of Vicq d'Azyr was not identified.

Other Parts of the Brain.-Large survey sections of representative parts of the cerebral cortex revealed no significant lesions. The corpus striatum, globus pallidus, and nucleus subthalamicus of each side were also normal. Myelination of the centrum semiovale and the basal ganglia was adequate for a child of this age but there was an abnormal fibrillary gliosis in the internal capsule, the medullary laminae of the globus pallidus, and in the optic tracts. The corpus callosum was normal.

\section{Case 2}

Clinical History.-J. A., a girl, was aged 8 months at death. There were three other siblings, all healthy, and the mother had had two miscarriages. The patient was born in the 36th week of pregnancy by Caesarian section on account of placenta praevia. The birth weight was $6 \mathrm{lb} .12 \mathrm{oz}$. The infant would not suckle and had to be fed by tube. After three months in hospital the weight was $8 \mathrm{lb}$. and she went home. Her health was precarious and before her terminal illness she had had four admissions to hospital for respiratory or urinary infections. At 18 weeks she was admitted with signs of pneumonia. At this time the circumference of the head was $15 \frac{1}{2}$ in. and the anterior fontanelle almost closed. She had made no intellectual progress and appeared to have no appreciation of light or sound. Spastic paresis was noted in the legs. Courses of penicillin and later of terramycin were given with little or no effect and death occurred from bronchopneumonia.

Examination of the Central Nervous System in Case 2

Macroscopic.-The brain after fixation weighed $513 \mathrm{~g}$., the cerebellum and brain-stem accounting for $51 \mathrm{~g}$.

The whole corpus callosum was absent and the cingulate gyri were ill defined. The left calcarine fissure failed to meet the parieto-occipital fissure. Both insulae were partly exposed owing to the poor development of the inferior frontal gyrus. The right superior frontal gyrus and both superior temporal gyri were unduly narrow. The cranial nerves and vessels at the base were normally distributed.

The cerebellum was smaller than normal and about $2 \mathrm{~cm}$. of the basal surface of the occipital lobes remained uncovered by it. The vermis, flocculi, and in particular the lateral lobes, were all hypoplastic and the brain-stem correspondingly small (Fig. 10a). The medullary pyramids were absent.

The right cerebral hemisphere was cut in coronal blocks (the left horizontally) and embedded in celloidin. The absence of the whole of the corpus callosum was confirmed and it was noted that no longitudinal callosal bundle was present on either side. The posterior and inferior horns of the lateral ventricles were enlarged owing to the poor development of the central white matter.

Microscopic.-In the cerebral cortex the granular layer of the visuo-sensory area was not divided by a stria of Gennari and contained no stellate cells. Elsewhere no gross abnormality was detected, though there was probably a mild generalized retardation in cortical development. The precentral gyrus contained numerous giant cells of Betz despite the absent pyramidal tracts. The whole of the hemispheric white matter showed a moderate fibrillary gliosis and in general the brain was rather less well myelinated than normal.

The thalamus, putamen, and caudate nucleus were histologically normal. The globus pallidus was unaffected except for its internal segment where the nerve cells were shrunken, some appearing pale, others staining deeply. There was marked fibrillary gliosis in this segment and also in the medullary laminae (Fig. 12) but this was not accompanied by detectable demyelination. The optic tracts were small and gliosed. The anterior commissure was present. Both the corpora Luysii were strikingly atrophied, and had lost diffusely about $90 \%$ of their nerve cells. The degeneration of the right corpus Luysii appeared to be the more recent since several figures of neuronophagia were seen (Fig. 11) and some of the remaining nerve cells appeared degenerate, with loss of Nissl bodies, swelling of the cytoplasm, and displacement of the nucleus to the margin of the cell. There was a well-marked fibrillary gliosis of the capsule and in the central part of the nucleus (Fig. 12) where the myelinated fibres were reduced in number.

Cerebellum.-There was no appreciable loss of Purkinje or granular cells in the lateral lobes or vermis and the central white matter was well myelinated. The dentate nuclei were small and poorly convoluted (Fig. 10a). Many of their nerve cells were shrunken and darkly staining, others were very small and almost devoid of Nissl substance. Here 
Fig. 10a.-Case 2. (a) Relatively small cerebellum and brain-stem. The dentate nucleus is poorly convoluted.

Heidenhain $\times 1.6$

Fig. 10b.-A normal control aged 9 months. Same magnification as $10(a)$.

Fig. 11.-Case 2. Left nucleus subthalamicus: severe neuronal degeneration. Several figures of neuronophagia are present.

Carbol azure $\times 70$

Fig. 12.-Case 2. Fibrillary gliosis of the nucleus subthalamicus and the globus pallidus (mainly the internal segment and medullary laminae).

Holzer $\times 5$

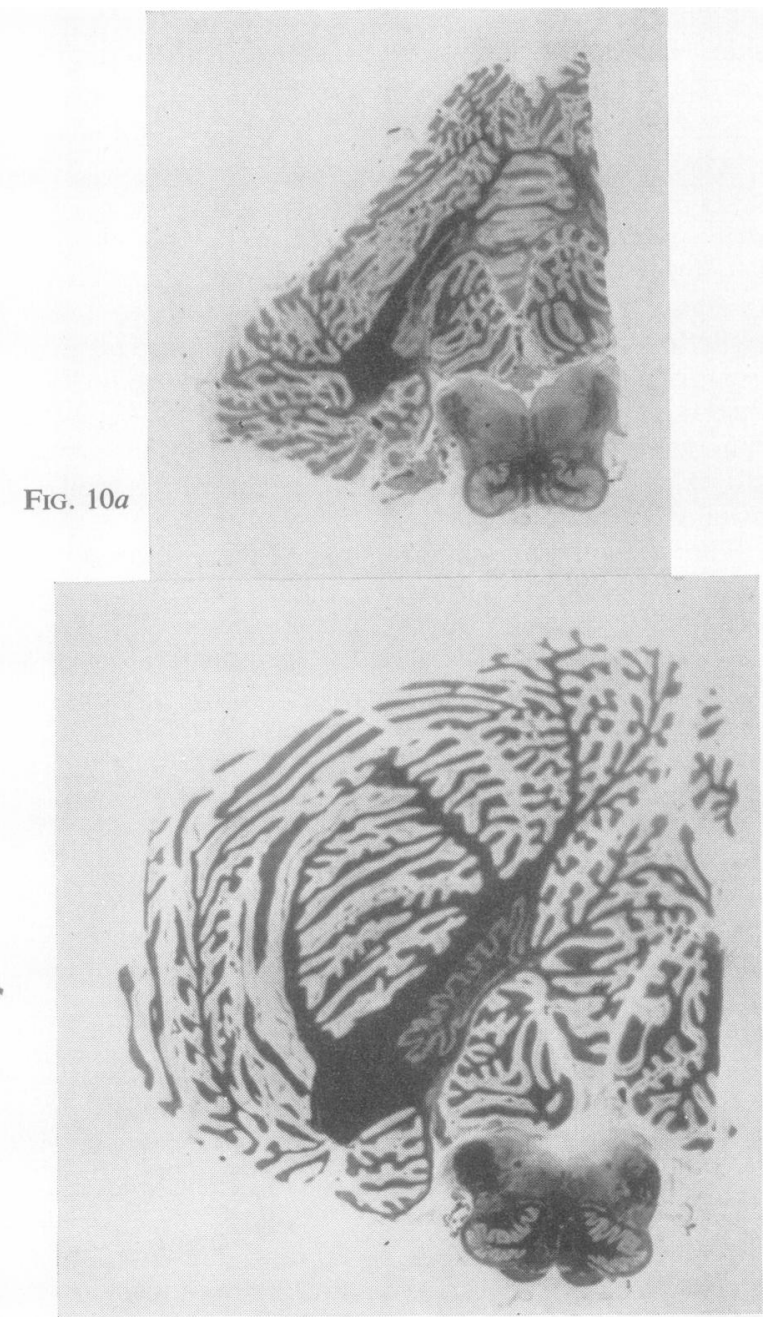

FIG. $10 b$
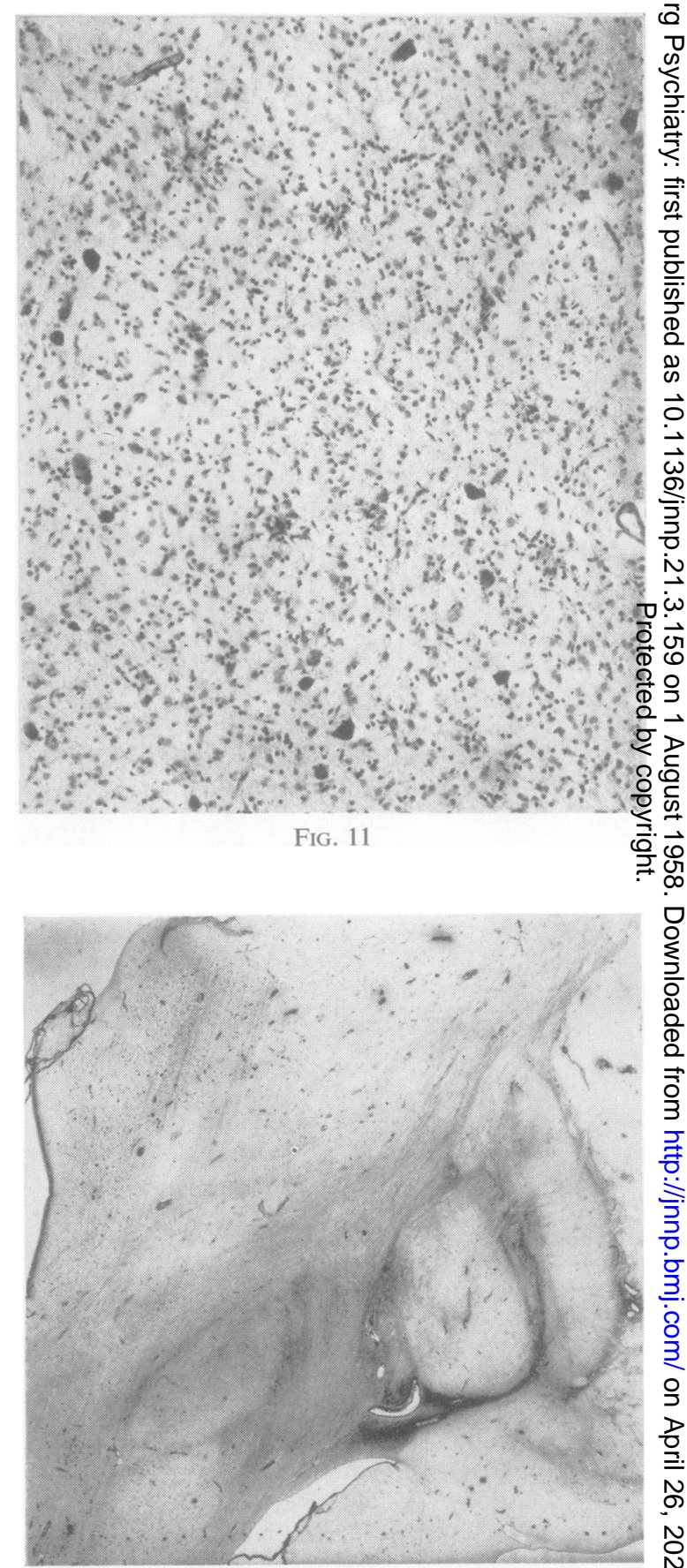

FIG. 12 
and there a patchy loss of nerve cells was obvious. The whole nucleus contained an increased number of astrocytes and in Holzer preparations the hilum showed a mild fibrillary gliosis. The nucleus emboliformis was similarly affected but the globosus and fastigii appeared normal on both sides.

Brain-stem.- The medullary pyramids were absent and the medial fillet poorly developed. The inferior olives showed no loss of nerve cells but caudally the cell bands were poorly convoluted. Serial sections failed to reveal any other abnormality in the nuclei of the brain-stem.

Spinal Cord.-Lateral fissures indicative of absent pyramidal tracts were present bilaterally in the cervical and dorsal segments. In the lumbar region the white matter showed a very dense fibrillary gliosis unassociated with detectable defect of myelination and not extending into the central grey matter.

\section{Discussion}

In comparing ponto-neocerebellar hypoplasia with olivo-ponto-cerebellar atrophy Brouwer cited the following structures as predominantly affected in both conditions: the lateral lobes of the cerebellum (Edinger's " neo-cerebellum "), the nuclei pont is and middle peduncle, the arcuate nucleus, and the corpus ponto-bulbare. The inferior olives, a major derivative of the migratory cell bands of Essick, have been found normal or but slightly underdeveloped in several examples of this form of cerebellar hypoplasia. However, in Brun's cases they showed a combination of hypoplasia and neuronal atrophy. Brun also noted a great reduction in the size of the lateral nuclei of the medulla and these also have been found to be degenerated in olivo-ponto-cerebellar atrophy (Winkler, 1923; van Bogaert and Bertrand, 1929; Tans, 1952). The detailed survey of the brain-stem made by Tans in a case of olivo-ponto-cerebellar atrophy has enabled us to elaborate this comparison between the two conditions. In our case, as in Tans', there were also obvious lesions in the perihypoglossal group of nuclei consisting of the nucleus intercalatus, the interfascicularis hypoglossi, and the nucleus of Roller. The nucleus conterminalis, the raphe obscurus, the paramedianus dorsalis, and the nuclei pararaphales were similarly underdeveloped or atrophied. Almost all these nuclei are known to have cerebellar connexions either in man (Uemura, 1917; Olszewski and Baxter, 1954) or in animals (Brodal, 1952). Such a close correspondence in the distribution of lesions cannot be due to chance. Nor is it probable that these brain-stem lesions are secondary to the cerebellar anomaly. Certainly, in olivo-ponto-cerebellar atrophy it is generally held that the pontine, arcuate, and olivary degenerations precede the very variable changes in the cerebellar cortex (Greenfield, 1954). While it is true that unilateral cerebellar hypoplasia may on occasion be associated with contralateral atrophy of olive or pons (Lichtenstein, 1943), this apparently retrograde type of atrophy seems to be the exception rather than the rule and may well indicate that some of the unilateral cases have a different pathogenesis from the bilateral. The brain-stem nuclei in our second case were intact despite the severe cerebellar hypoplasia. We are left with the conviction that Brouwer was justified in seeing the same principle of systemic degeneration in ponto-neocerebellar hypoplasia as in olivo-ponto-cerebellar atrophy. It becomes only a question of degree as to whether the congenital weakness of a neuronal system is so mild as to enable the nerve cells to survive for a number of years or so severe as to lead to atrophy in foetal life or even to a failure of cell division.

If this hypothesis is correct one would expect to find cases in which both prenatal malformations and unequivocal degenerative lesions occurred in the same brain, and in our first case degeneration and not hypoplasia involved the dentate nuclei. This finding is in contrast to previously reported examples of ponto-neocerebellar hypoplasia in which the dentate nucleus has shown a characteristic segmentation into several small islands of nerve cells, an appearance imitating that of the normal $80 \mathrm{~mm}$. embryo (Brun). In the present case, the clumps of nerve cells were evidently residual groups remaining after degeneration and gliosis of a normally convoluted nucleus. The almost complete atrophy of the ventro-lateral thalamic nuclei (in which the bulk of the superior cerebellar peduncles end) is a good example of chain degeneration and has recently been reported in abiotrophies involving the dentate nucleus (Poser, Dewulf, and Van Bogaert, 1957; Urich, Norman, and Lloyd, 1957). A further instance of chain degeneration and one which has no counterpart in diseases of later life was seen in the combined atrophy of mammillary bodies and anterior thalamic nuclei. This lesion is of special interest because of its evidently recent onset, as judged by the local microglial activity.

In the second case the dentate nuclei showed a combination of hypoplasia and atrophy. The most striking degenerative lesions were, however, found in the subthalamic nuclei where the presence of neuronophagia ruled out the possibility that these were lesions dating from the neonatal period. Once again cerebellar hypoplasia is seen in association with degenerative changes appropriate to the abiotrophies of later life (Titeca and van Bogaert, 
1946). It is logical to apply the same unifying principle of abiotrophy to the other malformations present in this case. Thus the absence of the pyramidal tracts and of the corpus callosum may be regarded not as the consequence of true agenesis but of early degeneration and resorption. As regards the absent corpus callosum, it is of considerable interest to note that there were no longitudinal callosal bundles. According to Marburg (1949), the absence of these structures, which are aberrant callosal fibres which have failed to cross the midline, is indicative of a destructive process affecting the corpus callosum after its formation. Provided this destruction occurred before the fifth month, the usual associated malformation of the cingulate gyrus might be expected to occur (Becker, 1952). In our case, therefore, it may be suggested that the degeneration and disappearance of the corpus callosum occurred at some period after its initial formation at the third month but before the cingulate convolution was laid down in the fifth month.

There was a strong hint of familial incidence in the first case and certainly the differing times of onset of the various lesions ranging from "pure" malformations to degenerations in active progress suggest the operation of genetic rather than exogenous factors. Clarification of this problem must await the collection of further examples of what at present seems a rare condition, namely, the combination of abiotrophy and prenatal malformation. All that is claimed for the apparently exceptional cases described here is that they form a bridge, albeit a slender one, connecting two categories of pathological process usually regarded as unrelated.

\section{Summary}

In two young infants cerebellar hypoplasia has been found associated with neuronal degenerative lesions of a systemic character. The first case was one of ponto-neocerebellar hypoplasia and showed, in addition to the usual developmental anomalies of the griseum pontis, arcuate nuclei, and inferior olives, the involvement of other brain-stem nuclei including the perihypoglossal and certain midline groups in the medulla. This distribution of lesions was remarkably similar in detail to that previously reported in olivo-ponto-cerebellar atrophy. Severe degeneration was present in the dentate nuclei and transneuronal atrophy had occurred in the ventrolateral nuclei of the thalamus. Another instance of chain degeneration was seen in the combined atrophy of the mammillary bodies and the antero-ventral thalamic nuclei. In the second case cerebellar hypo- $\vec{\Sigma}$ plasia was accompanied by absence of the corpus callosum and pyramidal tracts. The dentate nuclei $\stackrel{0}{\mathcal{D}}$ showed a combination of mild developmental arrest $\bar{C}$ and atrophy. Severe and recent degeneration was present in the corpora Luysii and this was associated with gliosis and neuronal shrinkage of the internal pallidal segments. These findings give support to of the theory first put forward by Brouwer that the same principle of systemic degeneration is to be seen in ponto-neocerebellar hypoplasia as in olivoponto-cerebellar atrophy. Some malformations may thus be the early expression of the same abiotrophic process that later reveals itself in the degeneration of certain vulnerable neuronal systems.

We are greatly indebted to Mr. G. L. Alexander an $\Phi \overrightarrow{ }$ to Dr. A. W. Franklin for the use of their clinical notes and also to Dr. R. J. Sandry and to Dr. J. I. Pugh whф్ 음 performed the post-mortem examinations and kindle made these brains available for examination.

This work was carried out with the help of the Nuffield Foundation.

\section{REFERENCES}

Becker, H. (1952). Disch. Z. Nervenheilk, 168, 345.

Biemond, A. (1955). Folia psychiat. (Amst.), 58, 2 .

Bogaert, L. van, and Bertrand, I (1929). Rev. neurol. (Paris), 1, 165.

Brodal, A. (1952). J. Anat. (Lond.), 86, 110.

Brouwer, B. (1924). Psychiat. neurol. Bl. (Amst.), 6, 461.

Brun, R. (1917). Schweiz. Arch. Neurol. Psychiat., 1, 61. (1918). Ibid., 2, 48.

Essick, C. R. (1912). Amer. J. Anat., 13, 25.

Greenfield, J. G. (1954). The Spino-cerebellar Degenerations. Blackwell, Oxford.

Lichtenstein, B. W. (1943). J. Neuropath., 2, 164.

Marburg O. (1949). Arch. Neurol. Psychiat. (Chicago) 61, 297.

Olszewski, J., and Baxter, D. (1954). Cytoarchitecture of the Human Brain Stem. Karger, New York.

Poser, C. M., Dewulf, A., and Bogaert, L. van (1957). J. Neuropath. $16,209$.

Tans, J. M. J. (1952). Proc. 1st International Congress of Neuropathology, Rome, Vol. 3, p. 478

Titica, J., and Bogaert, L. van (1946). Brain, 69, 251

Uemura, H. (1917). Schweiz. Arch. Neurol. Psychiat., 1, 151.

Urich, H., Norman, Schweiz. Arch. Neurol. Psychiat., 1, 151. (Basel), 17, 360 .

Vogt, H., and Astwazaturow, M. (1912). Arch. Psychiat. Nervenkr. 49, 75

Winkler, C. (1923). Schweiz. Arch. Neurol. Psychiat., 13, 684. 